\title{
Systemic Immune-Inflammatory Index as a Predictor of Lymph Node Metastasis in Endometrial Cancer
}

\author{
HuiFang Lei (D) ${ }^{1,2, *}$ \\ ShuXia Xu iD ${ }^{3, *}$ \\ XiaoDan Mao (D) ${ }^{1,2}$ \\ XiaoYing Chen iD ${ }^{4}$ \\ Yaojia Chen (iD) ${ }^{1,2}$ \\ XiaoQi Sun (iD) ${ }^{4}$ \\ PengMing Sun (D) 1,2,4 \\ 'Laboratory of Gynecologic Oncology, \\ Department of Gynecology, Fujian \\ Provincial Maternity and Children's \\ Hospital, Affiliated Hospital of Fujian \\ Medical University, Fuzhou, People's \\ Republic of China; ${ }^{2}$ Fujian Key \\ Laboratory of Women and Children's \\ Critical Diseases Research, Fujian \\ Provincial Maternity and Children's \\ Hospital, Affiliated Hospital of Fujian \\ Medical University, Fuzhou, People's \\ Republic of China; ${ }^{3}$ Department of \\ Pathology, Fujian Provincial Maternity and \\ Children's Hospital, Affiliated Hospital of \\ Fujian Medical University, Fuzhou, \\ People's Republic of China; ${ }^{4}$ Department \\ of Gynecology, Fujian Provincial \\ Maternity and Children's Hospital, \\ Affiliated Hospital of Fujian Medical \\ University, Fuzhou, People's Republic of \\ China
}

*These authors contributed equally to this work

Correspondence: XiaoQi Sun; PengMing Sun

Laboratory of Gynecologic Oncology,

Fujian Provincial Maternity and Children's Hospital, Affiliated Hospital of Fujian

Medical University, No. 18 Daoshan Road, Fuzhou, 35000I, Fujian, People's Republic of China

Email sunfemy@hotmail.com;

fmsun1975@fjmu.edu.cn
Purpose: This study assessed the predictive value of the preoperative systemic immuneinflammatory index (SII) for lymph node metastasis (LNM) in endometrial cancer (EC) patients.

Methods: We retrospectively included 392 EC patients between January 2013 and January 2019. Data on clinical indicators including age, body mass index (BMI), menopause, serum inflammatory immune index, serum tumor markers, history of diabetes and hypertension, stage, histological type, and myometrial invasion (MI) were collected. The association between clinical indicators and LNM was evaluated.

Results: The results indicated that neutrophil (NE), monocyte (MO) counts, SII, cancer antigen 125 (CA125), cancer antigen 153 (CA153), cancer antigen 199 (CA199), and the expression of estrogen receptor (ER) and Ki67 were higher in EC patients with LNM than in those without LNM $(\mathrm{P}<0.05)$. Lymph vascular space invasion (LVSI) was also associated with LNM $(\mathrm{P}<0.05)$. Consequently, the SII, CA125, CA153 and LVSI were found to be independent risk factors for LNM, and a nomogram including these indicators was performed. The ROC curve analysis showed that compared with a single index, the combination of the SII, CA125, CA153 and LVSI significantly improved the efficiency of diagnosing LNM in EC patients $(\mathrm{AUC}=0.865, \mathrm{P}<0.001)$. Moreover, the SII was significantly associated with age, menopause, and FIGO stage $(\mathrm{P}<0.05)$. Further logistic regression analysis suggested that elevated serum SII was an independent risk factor for MI and progression to a higher pathological grade in young premenopausal EC patients. In addition, elevated SII was an independent risk factor for advanced EC progression in age $\geq 55$ or postmenopausal EC patients.

Conclusion: An elevated SII is an independent risk factor for LNM in patients with EC. In addition, the SII can be used as a predictor of MI and higher pathological grade in young premenopausal EC patients.

Keywords: systemic immune-inflammatory index, neutrophil, platelet, lymphocyte count, lymph node metastasis, endometrial cancer

\section{Introduction}

Endometrial cancer (EC) is one of the most common malignant tumors of the female reproductive tract, with a lymph node metastasis (LNM) rate ranging from $10.5 \%$ to $14.9 \%{ }^{1-3}$ LNM is an important factor influencing the poor prognosis of EC patients, which not only affects the pathological stage of surgery but also may lead to serious consequences such as recurrence and distant metastasis, thus reducing the long-term survival rate of patients. ${ }^{3,4}$ Surgery is the preferred treatment for 
EC, and the basic surgical methods include cytological examination with retention of peritoneal fluid, laparoscopic extrafascial hysterectomy, bilateral adnexectomy, and abdominal aorta and pelvic lymph node dissection. ${ }^{4}$ However, the need for para-aortic and pelvic lymph node dissection in patients with low-risk and stage IA EC remains controversial. Some scholars believe that standardized lymph node dissection not only has auxiliary value in diagnosing LNM in patients with EC but also provides guidance for standardized postoperative treatment, which can reduce postoperative pelvic recurrence. ${ }^{5,6}$ However, some scholars believe that lymph node dissection does not improve the survival rate and tumor-free survival of patients but does cause lower limb pain, urinary fistula, bladder fistula and other postoperative complications in EC patients after surgical treatment. ${ }^{7-9}$ Therefore, preoperative overall evaluation is necessary. At present, imaging and tumor marker detection are the main methods for evaluating LNM in patients with EC. Imaging diagnosis is the clinical preoperative assessment of LNM in patients with EC, and the commonly used methods, such as ultrasonography, computerized tomography (CT), and magnetic resonance imaging (MRI), are affected by factors such as instruments and doctor experience. Hence, there is a certain probability of missed diagnosis and misdiagnosis. Cancer antigen 125 (CA125) is a tumor marker with good sensitivity closely related to the prognosis of EC, especially in EC patients with abdominal metastasis. ${ }^{10}$ Many studies have shown that CA125 can predict LNM in EC. ${ }^{11}$ However, the level of serum CA125 can increase to varying degrees, such as in endometriosis ${ }^{12}$ and hepatitis, ${ }^{13}$ which indicates that the specificity of CA125 as a tumor marker for EC is not high. Therefore, it is of great significance to actively seek scientific and effective LNM prediction methods for the formulation of treatment plans for patients with EC.

In recent years, the tumor microenvironment has received increasing attention, and a variety of inflammatory cells and inflammatory mediators are important components of the tumor microenvironment. Many studies have demonstrated that the systemic inflammatory response is related to the postoperative survival of tumor patients. ${ }^{14-17}$ Serum inflammatory factors can be detected in an easy and convenient way. Recently, the SII based on peripheral lymphocyte (Lym), neutrophil (NE) and platelet (PLT) counts has been considered a better index to reflect the local immune response and systemic inflammation, as its high prognostic value has been confirmed in a variety of tumors, such as cervical cancer, ${ }^{15}$ pancreatic cancer, ${ }^{16}$ and colorectal cancer. ${ }^{17}$ Moreover, the SII has also been shown to be closely associated with poor prognosis of EC. ${ }^{18,19}$ However, the relationship between the SII and LNM in EC patients remains unclear.

Therefore, this study evaluated the relationship between the SII and LNM in EC patients to identify a suitable indicator for clinicians to use when performing preoperative risk assessments of LNM in EC patients to aid in clinical diagnosis and treatment.

\section{Patients and Methods}

\section{Subjects}

The current study retrospectively included patients who underwent primary hysterectomy for EC at Fujian Provincial Maternity and Children's Hospital from January 2013 to January 2019 and had immunohistochemical pathological results.

The exclusion criteria included the following: 1) other malignant tumors or a history of other malignant tumors $(\mathrm{n}=3) ; 2)$ acute or chronic inflammation, immune disease, or hematologic disease $(n=4) ; 3)$ preoperative chemotherapy, radiotherapy or hormone therapy ( $\mathrm{n}=0)$; and 4$)$ loss to follow-up $(n=5)$. A total of 392 patients were included. We obtained informed consent from all the included patients. The Ethics Committee of Fujian Provincial Maternity and Children's Hospital approved the study.

\section{Data Collection and Definitions of Systemic Inflammatory Indexes}

Data on demographic and clinical indicators were collected from the Fujian Provincial Maternity and Children's Hospital information system. This information included age, body mass index (BMI), white blood cell (WBC), NE, monocyte (MO), PLT, Lym, T cell, B cell, CD4+T Lym, CD8+T Lym, and NK cell counts, cancer antigen 125 (CA125), cancer antigen 199 (CA199) and 153 (CA153), and carcinoma embryonic antigen (CEA) levels, menopausal status, history of diabetes and hypertension, International Federation of Gynecology and Obstetrics (FIGO) stage, histological type, tumor grade, myometrial invasion (MI), lymphovascular space invasion (LVSI), LNM, and the expression levels of estrogen receptor (ER), progesterone receptor (PR), and Ki67. Blood testing was carried out within 1 week before surgery.

The inflammatory indexes were calculated as preoperative inflammatory indicators with the following formulas: SII $=$ PLT count $\times$ NE count/Lym count. 


\section{Statistical Analysis}

Statistical analysis was performed with SPSS 22.0 (IBM Corp., Armonk, NY, USA) and R version 4.0.2. Continuous variables were analyzed by Student's $t$-tests or Mann-Whitney $U$-tests. Chi-square tests or Fisher's exact tests were used to analyze the categorical variables. A receiver operating characteristic (ROC) curve was generated for the cutoff point of the continuous data. The areas under the curve (AUCs) are provided with their sensitivity, specificity, and $95 \%$ confidence intervals (CIs). The significance of the obtained cutoff values associated with EC was tested by performing both univariate and multivariate binary logistic regression analyses. Adjusted risk estimates were obtained with logistic regression models and accounted for the variables used for matching. A nomogram was constructed via the rms $\mathrm{R}$ package.
Validation of the nomogram included calibration and discrimination. Calibration was evaluated by calibration plots and Hosmer-Lemeshow tests to calculate the consistency between the observed and predicted probabilities. A HosmerLemeshow $\mathrm{P}$ value $>0.05$ indicated good consistency. The discrimination-namely, the predictive accuracy of a nomogram - was evaluated by the ROC curve. Significance was set at $\mathrm{P} \leq 0.05$.

\section{Results}

\section{Baseline Characteristics}

Table 1 and Figure 1 show the clinical indicators associated with LNM in EC patients. The results indicated that serum inflammatory factors, including $\mathrm{NE}$ and $\mathrm{MO}$ counts and the SII, were higher in EC patients with

Table I Baseline Characteristics of EC Patients

\begin{tabular}{|c|c|c|c|}
\hline Parameter & No-LNM $(n=364)$ & $\operatorname{LNM}(n=28)$ & $\mathbf{P}$ \\
\hline Age, years & $54(50-59)$ & $55(47-61)$ & 0.714 \\
\hline $\mathrm{BMI}, \mathrm{kg} / \mathrm{m} 2$ & $24.14(22.29-26.66)$ & $24.38(21.88-26.30)$ & 0.959 \\
\hline $\mathrm{CA} 125, \mathrm{U} / \mathrm{L}$ & $18.45(12.25-32.20)$ & $54.30(24.40-136)$ & $<0.001$ \\
\hline CA199, U/L & 13.56(7.50-30.74) & $34.77(10.69-95.18)$ & 0.016 \\
\hline CAI53, U/L & $9.10(6.90-13.50)$ & $15.0(9.8-22.5)$ & $<0.001$ \\
\hline CEA, ng/mL & $1.81(1.22-2.73)$ & $1.75(1.20-2.67)$ & 0.928 \\
\hline \multicolumn{4}{|c|}{ Menopause status, n(\%) } \\
\hline Premenopausal & 174 & 9 & \\
\hline Postmenopausal & 190 & 19 & 0.110 \\
\hline \multicolumn{4}{|c|}{ History of diabetes, $n(\%)$} \\
\hline No & 282 & 22 & \\
\hline Yes & 82 & 6 & 0.893 \\
\hline \multicolumn{4}{|c|}{ History of hypertension, $n(\%)$} \\
\hline No & 216 & 18 & \\
\hline Yes & 148 & 10 & 0.608 \\
\hline \multicolumn{4}{|l|}{ LVSI } \\
\hline No-LVSI & 339 & 18 & \\
\hline LVSI & 25 & 10 & $<0.001$ \\
\hline \multicolumn{4}{|l|}{ ER expression } \\
\hline Low & 32 & 6 & \\
\hline High & 332 & 22 & 0.030 \\
\hline \multicolumn{4}{|l|}{ PR expression } \\
\hline Low & 44 & 7 & \\
\hline High & 319 & 21 & 0.052 \\
\hline Ki67(\%) & $40.0(22.5-60.0)$ & $50.0(40.0-70.0)$ & 0.007 \\
\hline
\end{tabular}

Note: $\mathrm{P}<0.05$ suggests significantly different.

Abbreviations: BMI, body mass index; CAI25, cancer antigen I25; CAI99, cancer antigen 199; CAI53, cancer antigen I53; CEA, a carcinoma embryonic antigen; LVSI, lymph vascular space invasion; ER, estrogen receptor; PR, progesterone receptor; ER/PR low expression included, ER/PR(-/ \pm ); ER/PR low expression included, ER/PR(+/++/+++). 
LNM than in those without LNM $(\mathrm{P}<0.05$, Figure 1$)$. In addition, the levels of serum CA125, CA153, and CA199 were elevated in the LNM group $(\mathrm{P}<0.05$, Table 1). Moreover, the expression of ER and Ki67 was higher in EC patients with LNM $(\mathrm{P}<0.05$, Table 1). LVSI was also associated with LNM $(\mathrm{P}<0.05$, Table 1). There were no differences observed between the EC patients with and without LNM with respect to age, BMI, WBC, Lym, PLT, T cell, B cell, CD4+ T cell, CD8 + T cell, NK cell counts, CEA level, menopausal status, history of diabetes and hypertension, or PR expression (all P > 0.05, Table 1, Figure 1).

\section{Independent Risk Factors for LNM in EC Patients}

The continuous data were dichotomized for subsequent analyses using ROC curve analysis and the Youden Index (Figure 2). The cutoff values for statistically significant LNM-related indicators, including NE, MO, SII, CA125, CA199, CA153, and Ki67, were identified. Moreover, the results of ROC curve analysis showed that these indicators had good predictive ability for LNM in EC (all $\mathrm{P}<0.05$, Figure 2). All baseline characteristics and clinicopathological features were analyzed by univariate and multivariate logistic regression analyses in EC
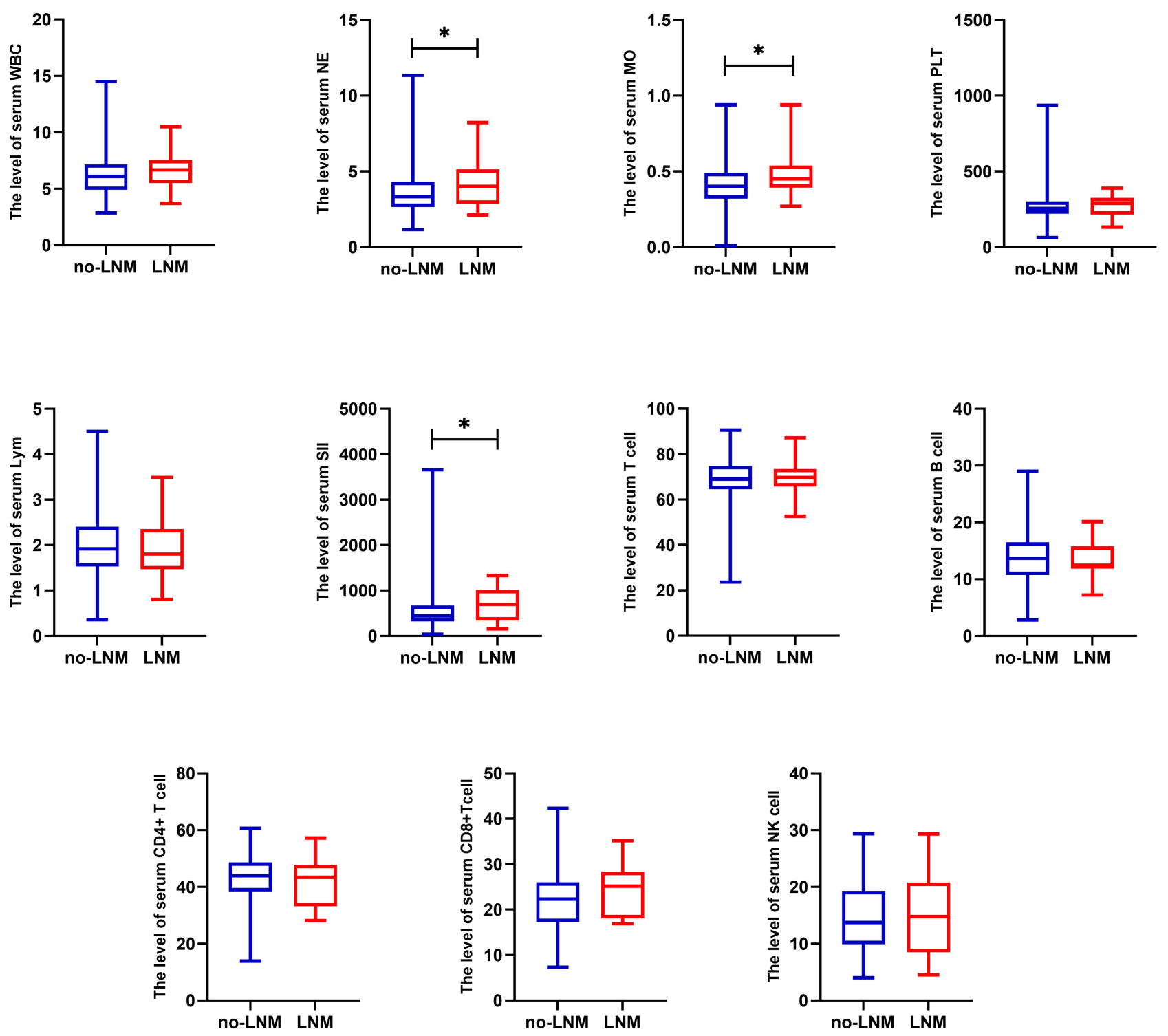

Figure I Characteristics of inflammatory immune cells in EC with LNM and no LNM.

Note: $* \mathrm{P}<0.05$. $\mathrm{P}<0.05$ indicates significant differences.

Abbreviations: WBC, white blood cell; NE, neutrophil; MO, monocyte; PLT, platelet count; Lym, lymphocyte; SII, systemic immune-inflammatory index; NK, natural killer. 

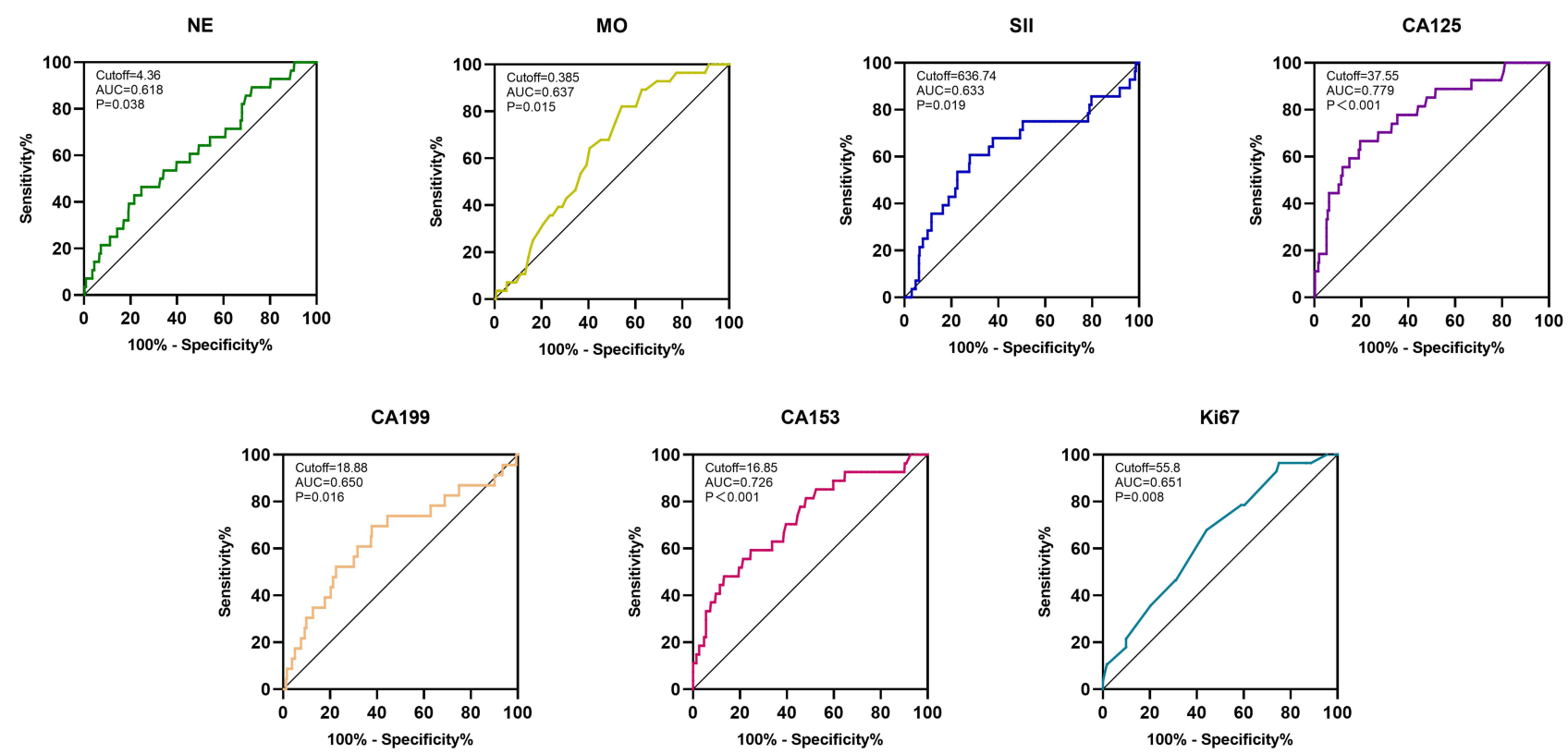

Figure 2 The ROC curve. The ROC curve for NE, MO, SII, CAI25, CA199, CAI53, and Ki67. The cutoff values for these indicators were identified. P<0.05 indicates significant differences.

Abbreviations: NE, neutrophil; MO, monocyte; SII, systemic immune-inflammatory index; CAI25, cancer antigen I25; CAI53, cancer antigen I53; CAI99, cancer antigen I99.

patients. The results of the univariate logistic regression analysis showed that $\mathrm{NE}$ and MO counts, the SII, the levels of CA125, CA153, and CA199, and LVSI and ER expression were significantly associated with LNM (all $\mathrm{P}<0.05$, Figure 3A). Then, significant factors from the univariate analysis were included in the multivariate analysis. The results showed that the SII, CA125, CA153 and LVSI were independent risk factors for LNM (all $\mathrm{P}<0.05$, Figure 3B). The significant independent predictors identified in the logistic regression analysis were used to construct a nomogram for LNM to provide a method for quantitative prediction (Figure 4A). Calibration plots and ROC curves were used to assess the predictive accuracy of the nomogram. The calibration plots graphically showed good consistency between the actual observations and the predicted probabilities in the prediction of LNM, with Hosmer-Lemeshow $\mathrm{P}=0.288$ (Figure 4B). The ROC curve analysis showed that compared with a single index, the combination of the SII, CA25, CA153 and LVSI could significantly improve the efficiency of diagnosing LNM in EC patients (AUC $=0.865, \mathrm{P}<0.001$, Figure 4B).

\section{Correlations Between Clinicopathological Features and the SII}

The SII was significantly associated with age, menopause, FIGO stage, and LNM (all P $<0.05$, Table 2). To further explore the clinical application value of the SII, we analyzed EC patients according to age and menopause. The results showed that the SII was closely related to pathological grade and $\mathrm{MI}$ in young premenopausal EC patients (all $\mathrm{P}<0.05$, Table 3). In EC patients age $\geq 55$ years or with postmenopausal EC, the SII was associated with FIGO stage (all $\mathrm{P}<0.05$, Table 3). Further logistic regression analysis suggested that an elevated serum SII was an independent risk factor for MI and progression to a higher pathological grade in young premenopausal EC patients ( $\mathrm{P}<0.05$, Figure $5 \mathrm{~A}$ and $\mathrm{B})$. In addition, an elevated SII was an independent risk factor for advanced EC progression in age $\geq 55$ or postmenopausal EC patients $(\mathrm{P}<0.05$, Figure $5 \mathrm{C})$.

\section{Discussion}

LNM is the main manifestation of EC metastasis and a key factor affecting the prognosis of patients with EC. Pelvic LNM is closely related to the prognosis of patients with EC, which is of great significance for the formulation of clinical treatment plans and prognosis monitoring of patients with EC. ${ }^{3,4}$ At present, there is no effective serum marker to predict LNM in EC patients. In previous studies, the systemic inflammatory response has been proven to be a factor in the poor prognosis of patients with various cancers. ${ }^{15-19}$ To investigate the simple and effective prognostic indexes used in the evaluation of the prognosis and guidance of clinical treatment of EC patients, many indexes based on 


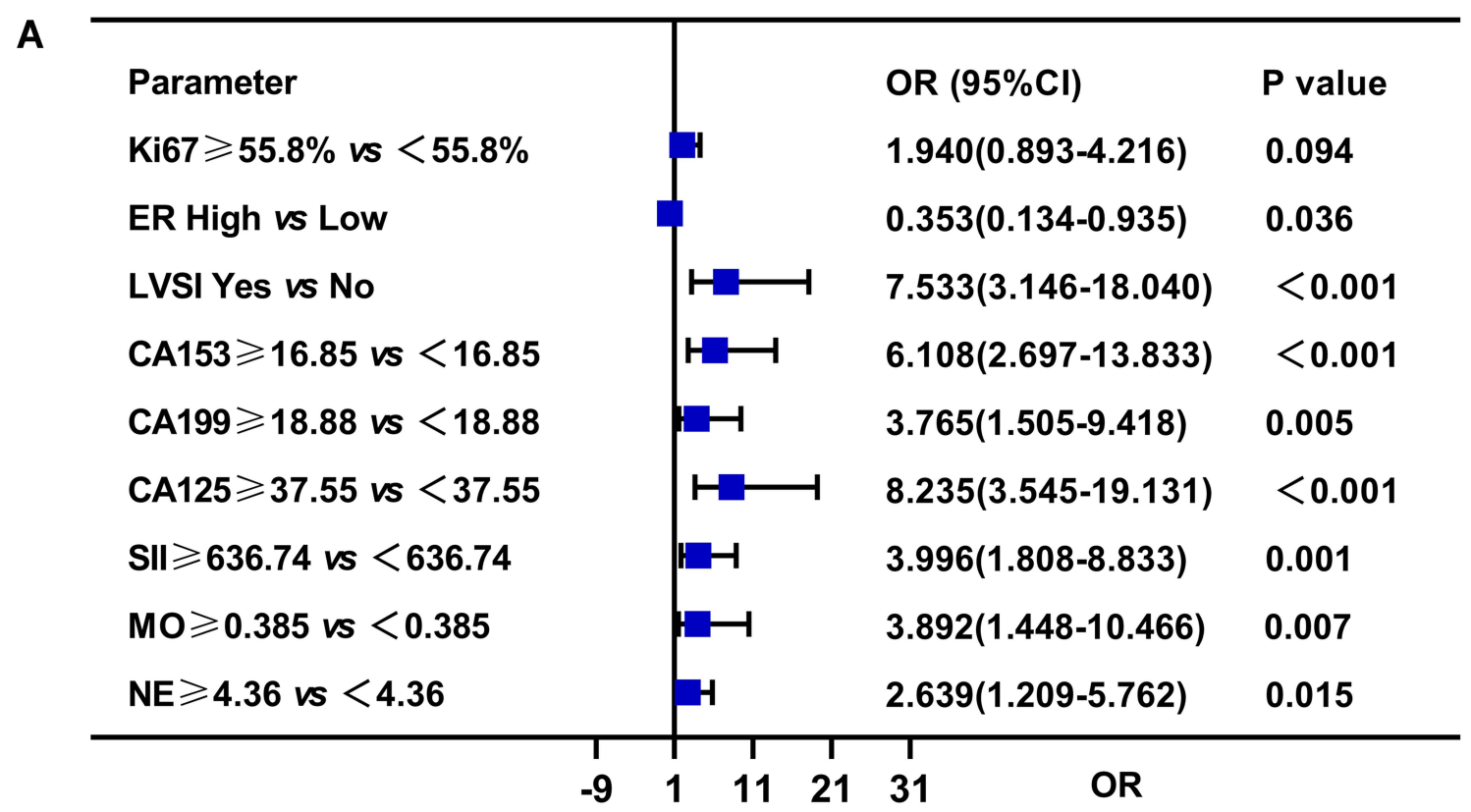

\begin{tabular}{|c|c|c|}
\hline Parameter & OR $(95 \% \mathrm{Cl})$ & $P$ value \\
\hline ER High vs Low & $0.581(0.158-2.138)$ & 0.414 \\
\hline LVSI Yes vs No & $4.518(1.370-14.904)$ & 0.013 \\
\hline CA153 $\geqslant 16.85$ vs $<16.85$ & $3.877(1.322-11.374)$ & 0.014 \\
\hline CA199 $\geqslant 18.88$ vs $<18.88$ & $1.874(0.632-5.554)$ & 0.257 \\
\hline CA125 $\geqslant 37.55$ vs $<37.55$ & $4.309(1.479-12.555)$ & 0.007 \\
\hline$S I I \geqslant 636.74$ vs $<636.74$ & $4.689(1.334-16.476)$ & 0.016 \\
\hline $\mathrm{MO} \geqslant 0.385$ vs $<0.385$ & $3.176(0.997-10.120)$ & 0.051 \\
\hline $\mathrm{NE} \geqslant 4.36$ vs $<4.36$ & $0.946(0.285-3.139)$ & 0.928 \\
\hline
\end{tabular}

Figure 3 The logistic regression analyses. (A) Univariate logistic regression analyses for LNM. (B) Multivariate logistic regression analyses for LNM. P<0.05 indicates significant differences.

inflammation were studied and discussed, and this exploration focused on the value of the SII in the prediction of LNM in EC patients. Our study is the first to suggest that an elevated SII is an independent risk factor for LNM in patients with EC. In addition, the SII has different application value in pre- and postmenopausal EC patients. In premenopausal patients with EC, the SII can be used to assist in the assessment of deep MI and higher pathological grade. The results have implications for young women who want to keep their uterus if they have a reproductive need. In postmenopausal EC patients, the SII was more closely associated with FIGO stage. An elevated SII was an independent risk factor for advanced EC in postmenopausal patients.

The inflammatory immune microenvironment plays an important role in tumorigenesis, development and metastasis. ${ }^{20}$ Changes in the inflammatory tumor microenvironment contribute to the acquisition of malignant characteristics such as cancer cell proliferation, 
A

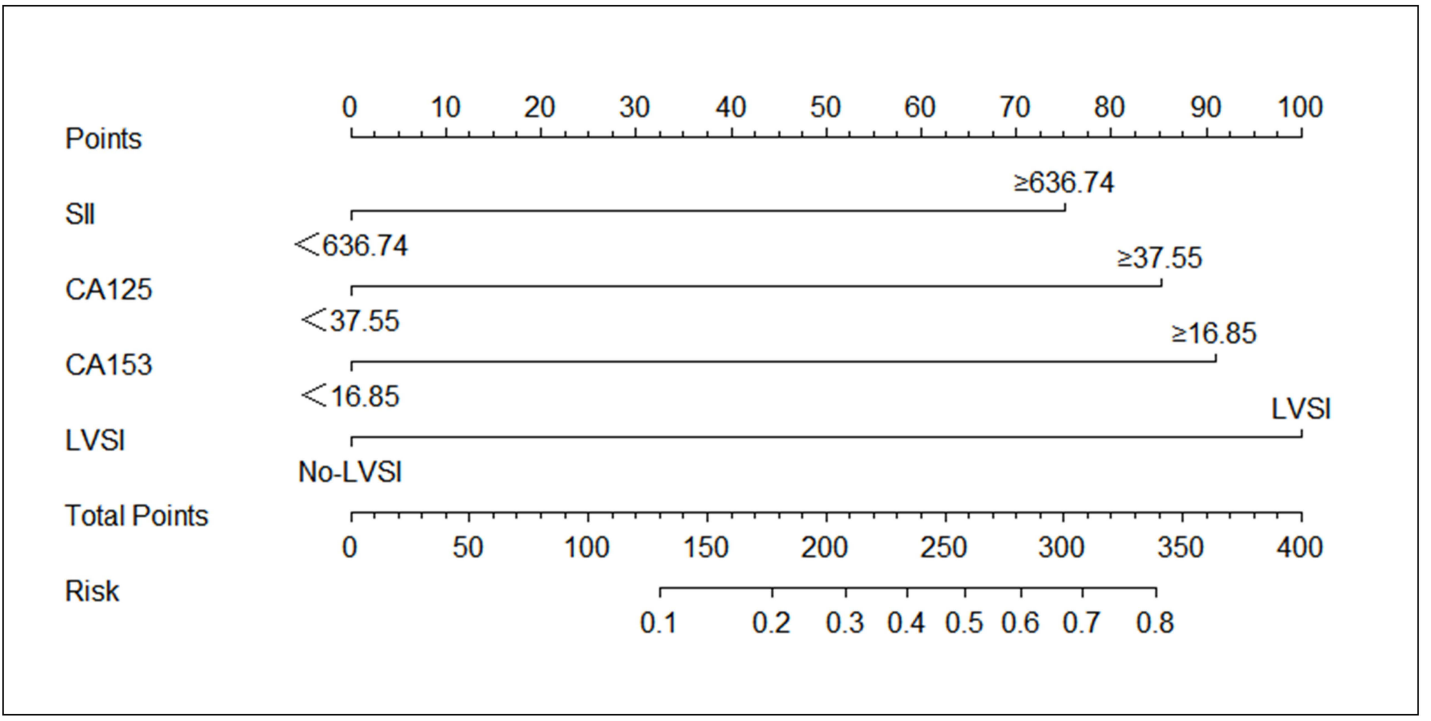

B

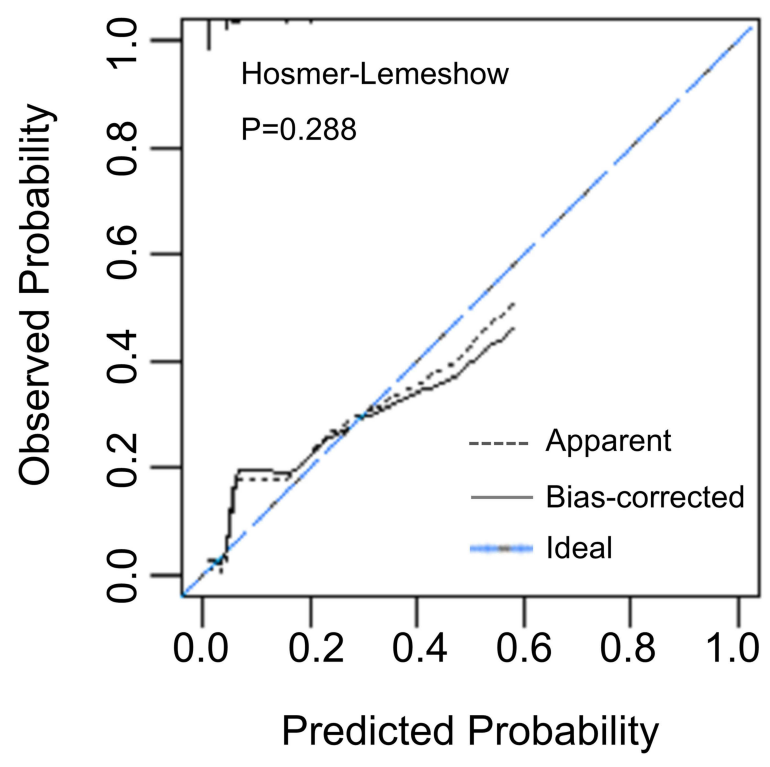

C

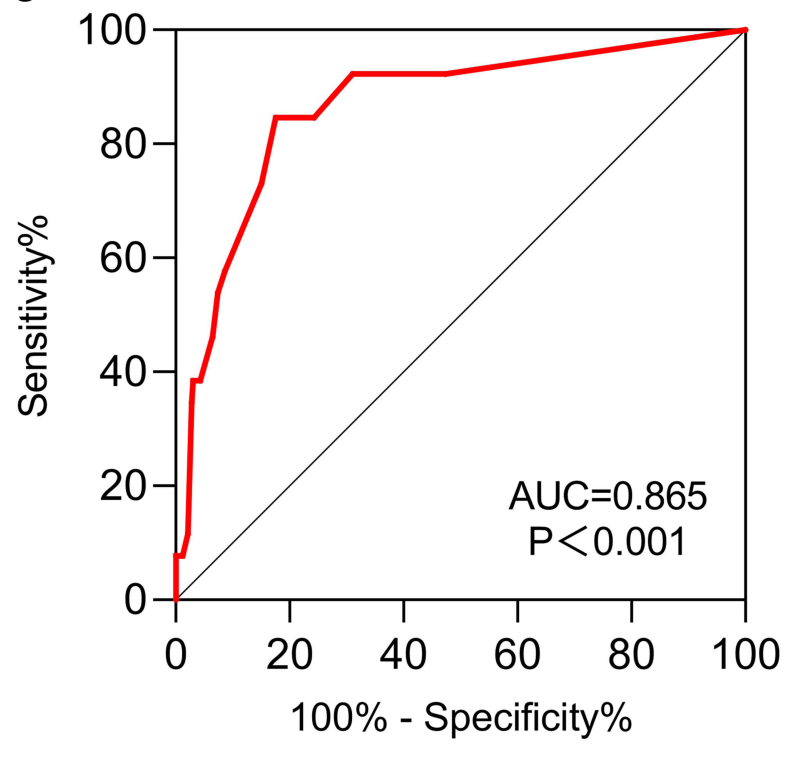

Figure 4 (A) The nomogram for predicting LNM with SII, CAI25, CAI53, and LVSI. (B) The calibration plots for the nomogram. (C) The ROC curve for the nomogram.

aggressiveness, metastasis, angiogenesis, and immune escape. $^{20,21}$ Common inflammatory cells in peripheral blood include WBCs, Lyms, NEs, MOs and PLTs. The relationship between systemic inflammatory markers and tumors has become a current research hotspot.

Lyms have an immune recognition function and are an important part of the immune response mechanism of the body. Lyms are involved in the regulation of tumor immunity and play a role in killing tumor cells by establishing an immune barrier. ${ }^{22,23}$ A decrease in the Lyms level means that the immune function of the body is weakened and the antitumor immune ability is decreased, thus promoting the recurrence and metastasis of tumors. ${ }^{22,23}$ However, studies on Lym counts in EC patients are limited. Some studies have shown that the Lym count is associated with the prognosis of EC. ${ }^{24}$ In contrast, some studies suggest that the Lym count does not play a significant role in EC prognosis assessment. ${ }^{25}$ In our study, there was no significant difference in the Lym counts among the EC patients with or without LNM. The differences in these results may be due to population differences or individual Lym counts that may be greatly influenced by changes in the body environment. 
Table 2 Associations of SII with Clinicopathological Characteristics

\begin{tabular}{|c|c|c|c|c|}
\hline Parameter & $\begin{array}{c}S I I<636.74 \\
(n=267)\end{array}$ & $\begin{array}{c}\text { SII } \geq 636.74 \\
(n=I \mid 6)\end{array}$ & $X^{2}$ & $\mathbf{P}$ \\
\hline \multicolumn{5}{|l|}{ Age, $n(\%)$} \\
\hline$<55$ years & $|4|$ & 76 & & \\
\hline$\geq 55$ years & 126 & 40 & 5.318 & 0.021 \\
\hline \multicolumn{5}{|l|}{ BMI, n(\%) } \\
\hline$<25 \mathrm{~kg} / \mathrm{m} 2$ & 142 & 58 & & \\
\hline$\geq 25 \mathrm{~kg} / \mathrm{m} 2$ & 90 & 46 & 0.881 & 0.349 \\
\hline \multicolumn{5}{|c|}{ Menopause status, $\mathrm{n}(\%)$} \\
\hline Premenopausal & 111 & 67 & & \\
\hline Postmenopausal & 156 & 49 & 8.516 & 0.004 \\
\hline \multicolumn{5}{|l|}{ FIGO stage, n(\%) } \\
\hline I-II & 237 & 92 & & \\
\hline III-IV & 25 & 20 & 5.125 & 0.024 \\
\hline \multicolumn{5}{|l|}{ Histologic type, n(\%) } \\
\hline EEC & 233 & 97 & & \\
\hline NEEC & 34 & 19 & 0.901 & 0.339 \\
\hline \multicolumn{5}{|c|}{ Histologic grade, n(\%) } \\
\hline Gradel/2 & 209 & 81 & & \\
\hline Grade 3 & 22 & 13 & 1.289 & 0.257 \\
\hline \multicolumn{5}{|c|}{ Myometrial invasion, n(\%) } \\
\hline$<50 \%$ & 168 & 62 & & \\
\hline$\geq 50 \%$ & 97 & 54 & 3.337 & 0.068 \\
\hline \multicolumn{5}{|l|}{ LVSI, n(\%) } \\
\hline No & 246 & 102 & & \\
\hline Yes & 21 & 14 & 1.721 & 0.190 \\
\hline \multicolumn{5}{|l|}{ LNM, n(\%) } \\
\hline No & 256 & 99 & & \\
\hline Yes & 11 & 17 & 13.246 & $<0.001$ \\
\hline \multicolumn{5}{|l|}{ ER expression, $n(\%)$} \\
\hline Low & 22 & 16 & & \\
\hline High & 245 & 100 & 2.791 & 0.095 \\
\hline \multicolumn{5}{|l|}{ PR expression, $n(\%)$} \\
\hline Low & 32 & 18 & & \\
\hline High & 234 & 98 & 0.863 & 0.353 \\
\hline \multicolumn{5}{|c|}{ Ki67 expression, n(\%) } \\
\hline$<55.8 \%$ & 176 & 79 & & \\
\hline$\geq 55.8 \%$ & 82 & 35 & 0.043 & 0.836 \\
\hline
\end{tabular}

Note: $\mathrm{P}<0.05$ suggests significantly different.

Abbreviations: BMI, body mass index; FIGO, International Federation of Gynecology and Obstetrics; EEC, Endometrioid endometrial adenocarcinoma; NEEC, nonendometrioid endometrial cancer; LVSI, lymph vascular space invasion; ER, estrogen receptor; PR, progesterone receptor; SII, systemic Immune-Inflammatory Index; ER/PR low expression included, ER/PR(-/ \pm ); ER/PR low expression included, ER/PR(+/++/+++). 
Table 3 Correlations Between Clinical or Biochemical Parameters and SII in EC Patients

\begin{tabular}{|c|c|c|c|c|c|c|c|}
\hline \multicolumn{2}{|l|}{ Parameter } & \multicolumn{3}{|c|}{$\begin{array}{c}\text { Premenopausal and Age }<55 \\
\qquad(n=160)\end{array}$} & \multicolumn{3}{|c|}{$\begin{array}{l}\text { Postmenopausal or Age } \geq 55 \\
\qquad(n=232)\end{array}$} \\
\hline & & \multirow{2}{*}{$\begin{array}{c}\text { SII } \\
<636.74 \\
56\end{array}$} & \multirow{2}{*}{$\begin{array}{c}\text { SII } \\
\geq 636.74 \\
29\end{array}$} & $\mathbf{P}_{\mathbf{I}}$ & \multirow{2}{*}{$\begin{array}{c}\text { SII } \\
<636.74 \\
86\end{array}$} & \multirow{2}{*}{$\begin{array}{c}\text { SII } \\
\geq 636.74 \\
29\end{array}$} & \multirow[t]{2}{*}{$\mathbf{P}_{2}$} \\
\hline BMI & $<25$ & & & & & & \\
\hline & $\geq 25$ & 26 & 27 & 0.051 & 64 & 19 & 0.707 \\
\hline \multirow[t]{2}{*}{ FIGO stage } & I-II & 84 & 52 & & 153 & 40 & \\
\hline & III-IV & 9 & 9 & 0.339 & 16 & 11 & 0.021 \\
\hline Histologic & $\mathrm{ECC}$ & 89 & 56 & & 144 & 41 & \\
\hline type & NECC & 5 & 6 & 0.300 & 29 & 13 & 0.228 \\
\hline Histologic & Gradel/2 & 80 & 44 & & 129 & 37 & \\
\hline grade & Grade 3 & 4 & 8 & 0.034 & 18 & 5 & 0.953 \\
\hline Myometrial & $<1 / 2$ & 68 & 36 & & 100 & 26 & \\
\hline invasion & $\geq 1 / 2$ & 24 & 26 & 0.040 & 73 & 28 & 0.214 \\
\hline \multirow[t]{2}{*}{ LVSI } & No & 89 & 57 & & 157 & 45 & \\
\hline & Yes & 5 & 5 & 0.495 & 16 & 9 & 0.129 \\
\hline ER & Low & 6 & 6 & & 16 & 10 & \\
\hline expression & High & 88 & 56 & 0.451 & 157 & 44 & 0.062 \\
\hline PR & Low & 5 & 6 & & 27 & 12 & \\
\hline expression & High & 89 & 56 & 0.300 & 145 & 42 & 0.269 \\
\hline Ki67 & Low & 72 & 47 & & 104 & 32 & \\
\hline expression & High & 20 & 13 & 0.992 & 62 & 22 & 0.657 \\
\hline
\end{tabular}

Note: $\mathrm{P}<0.05$ suggests significantly different.

Abbreviations: BMI, body mass index; FIGO, International Federation of Gynecology and Obstetrics; LVSI, lymph vascular space invasion; ER, estrogen receptor; PR, progesterone receptor; SII, Systemic Immune-Inflammatory Index; ER/PR low expression included, ER/PR(-/ \pm ); ER/PR low expression included, ER/PR(+/++/+++).

NEs are effector cells of the acute inflammatory response and play a major role in the clearance of extracellular pathogens. These cells are involved in the activation, regulation and effector functions of innate and adaptive immune cells. ${ }^{26}$ Neutrophil elastase and vascular endothelial growth factor are closely related to tumor growth and metastasis. ${ }^{26}$ Moreover, when systemic inflammation occurs in the body, in addition to an increase in the number of NEs and a decrease in the number of Lyms, PLT levels increase, various chemokines activate PLTs, and activated PLTs release growth factors to support tumor growth and invasion. ${ }^{27}$ Chen et al confirmed that a high PLT count was independently associated with poor RFS and OS in EC patients. ${ }^{28}$ In addition, as an elevated SII reflects a status of elevated NE and PLT counts, we believe that factors that stimulate granulopoiesis and/or thrombopoiesis might also be involved in the mechanism responsible for the elevated SII in EC patients.

The SII is a novel index combining the counts of three inflammatory immune cells: Lyms, NEs and PLTs. Studies have shown that the SII can predict the survival prognosis of patients with a variety of tumors. ${ }^{15-19}$ Screening and identifying high-risk patients have application value.
Holub et $\mathrm{al}^{19}$ suggested that the SII is associated with poorer outcomes in surgically staged I-III FIGO EC patients classified as high risk and treated with adjuvant EBRT and could be considered at cancer diagnosis. Matsubara et $\mathrm{al}^{18}$ confirmed that the SII is an independent prognostic factor in EC patients, allowing more precise survival estimation than PLR or NLR. However, the relationship between the SII and LNM in EC remains unclear. In our study, an elevated SII was confirmed to be an independent risk factor for LNM in patients with EC and could be used as a serum predictor of LNM in patients with EC, with good clinical application value. In addition, the SII was significantly associated with age and menopause in the correlation analysis. Therefore, we divided the EC patients into two groups according to age and menopause for subgroup analysis. The results showed that in young premenopausal women, the SII was closely associated with MI and a higher pathological grade in EC. For young premenopausal EC patients with reproductive needs, it is of great significance to evaluate the occurrence of muscular infiltration and pathological grading status. These findings have not been reported previously. We consider that future investigation of the underlying 
A

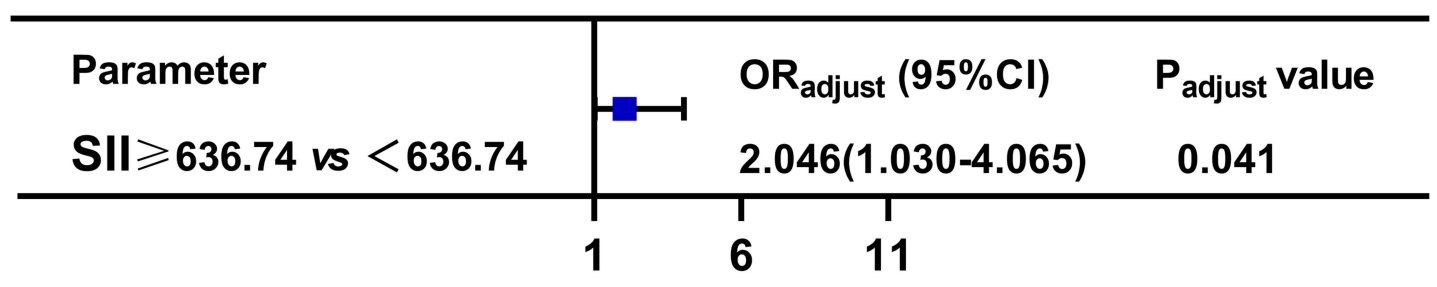

B

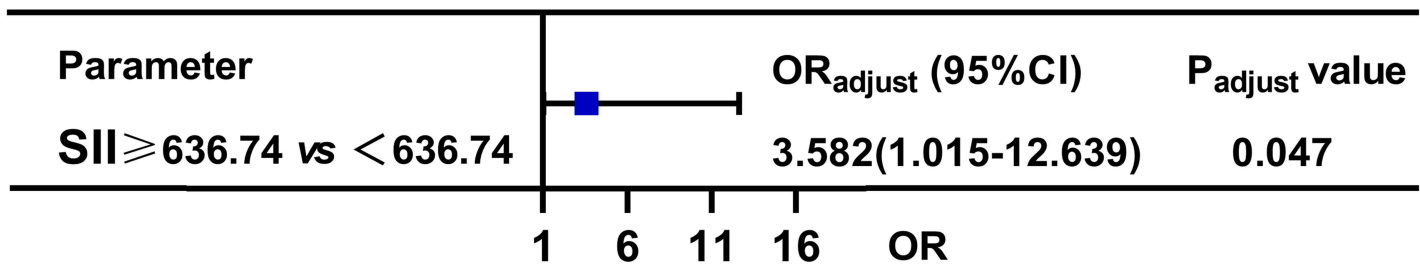

C

\begin{tabular}{|c|c|c|}
\hline Parameter & OR adjust $(95 \% \mathrm{Cl})$ & $\mathbf{P}_{\text {adjust }}$ value \\
\hline$S I I \geqslant 636.74$ vs $<636.74$ & 2.713(1.159-6.349) & 0.021 \\
\hline
\end{tabular}

Figure 5 The logistic regression analyses. (A) Multivariate logistic regression analyses for myometrial invasion in premenopausal young EC. (B) Multivariate logistic regression analyses for histologic grade in premenopausal young EC. (C) Multivariate logistic regression analyses for FIGO stage in postmenopausal or age $\geq 55$ EC.

Note: $P<0.05$ indicates significant differences.

Abbreviations: SII, Systemic Immune-Inflammatory Index; FIGO, International Federation of Gynecology and Obstetrics; OR ${ }_{\text {adjust }}$ OR adjust for age; $P_{\text {adjust }}$ P value adjusted for age.

causative mechanism of an elevated SII will aid in the development of novel effective treatments for EC.

LVSI is a high-risk factor for LNM in EC patients. ${ }^{29}$ The presence of LVSI is associated with LNM, a critical factor with regard to staging, prognosis and treatment planning of clinically early-stage EC patients. ${ }^{30}$ Consistent with previous studies, our results suggest that LVSI is an independent risk factor for LNM in EC patients. However, as LVSI is mostly diagnosed by pathology after surgery, it cannot be evaluated before surgery. Our study, combined with serum indicators, is easy to detect and can be used as an auxiliary indicator to determine LNM in patients with EC, with good clinical application value.

In addition, CA125 and CA153 were confirmed to be independent risk factors for LNM in EC patients in our study, which was consistent with previous studies. ${ }^{31-33}$ Moreover, our study combined the SII, CA125, CA153 and LVSI to significantly improve the efficacy of predicting LNM in EC patients.
There were also some limitations to this study. First, selection bias may exist, as this is a single-center retrospective study that represents the population only in some regions of China. Future studies should include a large, multicenter sample. Second, peripheral blood cell analysis results are easily affected by factors such as blood circulation capacity, infection, and nutritional status. Moreover, the treatment of patients after surgical resection has some heterogeneity, leading to different clinical outcomes.

In conclusion, the results of our study confirm that an elevated SII is an independent risk factor for LNM in patients with EC. In addition, the SII has different clinical application value in EC patients according to age and menopausal status. Notably, the SII can be used as a predictor of muscle invasion and a higher pathological grade in young premenopausal EC patients. 


\section{Data Sharing Statement}

The datasets generated and/or analyzed during the current study are available from the corresponding author on reasonable request.

\section{Ethical Approval}

This study was approved by the ethical committee of the Department of Gynecology of Fujian Provincial Maternity and Children's Hospital. The study complied with the Declaration of Helsinki.

\section{Author Contributions}

HuiFang Lei and ShuXia Xu contributed to the conception and design of the study; to the acquisition, analysis, and interpretation of data; and to writing the article. XiaoDan Mao and XiaoYing Chen contributed to the statistical analysis. YaoJia Chen contributed to the methods and performed the laboratory analyses. PengMing Sun and XiaoQi Sun contributed to the acquisition of data, critically revised the article for important intellectual content, and supervised the study. All authors made a significant contribution to the work reported, whether that is in the conception, study design, execution, acquisition of data, analysis and interpretation, or in all these areas; took part in drafting, revising or critically reviewing the article; gave final approval of the version to be published; have agreed on the journal to which the article has been submitted; and agree to be accountable for all aspects of the work.

\section{Funding}

Natural Science Foundation of Fujian Province (2021J01404).

\section{Disclosure}

The authors declare that they have no conflicts of interest.

\section{References}

1. Lee J, Kong T, Paek J, Chang S, Ryu H. Predicting model of lymph node metastasis using preoperative tumor grade, transvaginal ultrasound, and serum CA-125 level in patients with endometrial cancer. Int $J$ Gynecol Cancer. 2016;26(9):1630-1635. doi:10.1097/ IGC.0000000000000820

2. Karalok A, Turan T, Basaran D, et al. Lymph node metastasis in patients with endometrioid endometrial cancer: overtreatment is the main issue. Int J Gynecol Cancer. 2017;27(4):748-753. doi:10.1097/ IGC.0000000000000937

3. Sari M, Yalcin İ, Sahin H, Meydanli M, Gungor T. Risk factors for paraaortic lymph node metastasis in endometrial cancer. Int $J$ Clin Oncol. 2017;22(5):937-944. doi:10.1007/s10147-017-1139-5
4. Creasman W. Revised FIGO staging for carcinoma of the endometrium. Int $J$ Gynaecol Obstetr. 2009;105(2):109. doi:10.1016/j.ijgo.2009.02.010

5. Amant F, Mirza M, Koskas M, Creutzberg C. Cancer of the corpus uteri. Int $J$ Gynaecol Obstetr. 2018;143:37-50. doi:10.1002/ ijgo. 12612

6. Geisler J, Linnemeier G, Manahan K. Pelvic and para-aortic lymphadenectomy in patients with endometrioid adenocarcinoma of the endometrium. Int $J$ Gynaecol Obstetr. 2007;98(1):39-43. doi:10.1016/j.ijgo.2007.03.035

7. Benedetti Panici P, Basile S, Maneschi F, et al. Systematic pelvic lymphadenectomy vs. no lymphadenectomy in early-stage endometrial carcinoma: randomized clinical trial. $J$ Natl Cancer Inst. 2008;100(23):1707-1716. doi:10.1093/jnci/djn397

8. Todo Y, Sakuragi N. Methodological considerations in the analysis of the therapeutic significance of lymphadenectomy in endometrial cancer. Taiwan J Obstet Gynecol. 2013;52(1):8-13. doi:10.1016/j. tjog.2013.01.003

9. Cragun J, Havrilesky L, Calingaert B, et al. Retrospective analysis of selective lymphadenectomy in apparent early-stage endometrial cancer. J Clin Oncol. 2005;23(16):3668-3675. doi:10.1200/JCO.2005.04.144

10. Ünsal M, Kimyon Comert G, Karalok A, et al. The preoperative serum CA125 can predict the lymph node metastasis in endometrioid-type endometrial cancer. Ginekol Pol. 2018;89 (11):599-606. doi:10.5603/GP.a2018.0103

11. Yang B, Shan B, Xue X, et al. Predicting lymph node metastasis in endometrial cancer using serum CA125 combined with immunohistochemical markers PR and Ki67, and a comparison with other prediction models. PLoS One. 2016;11(5):e0155145. doi:10.1371/ journal.pone. 0155145

12. Kokot I, Piwowar A, Jędryka M, Sołkiewicz K, Kratz E. Diagnostic significance of selected serum inflammatory markers in women with advanced endometriosis. Int $J$ Mol Sci. 2021;22(5):2295. doi:10.3390/ijms22052295

13. Qin C, Gao Y, Li J, Huang C, He S. Predictive effects of preoperative serum CA125 and AFP levels on post-hepatectomy survival in patients with hepatitis B-related hepatocellular carcinoma. Oncol Lett. 2021;21(6):487. doi:10.3892/ol.2021.12748

14. Liu J, Li S, Zhang S, et al. Systemic immune-inflammation index, neutrophil-to-lymphocyte ratio, platelet-to-lymphocyte ratio can predict clinical outcomes in patients with metastatic non-small-cell lung cancer treated with nivolumab. J Clin Lab Anal. 2019;33(8):e22964. doi: $10.1002 /$ jcla. 22964

15. Huang H, Liu Q, Zhu L, et al. Prognostic value of preoperative systemic immune-inflammation index in patients with cervical cancer. Sci Rep. 2019;9(1):3284. doi:10.1038/s41598-019-39150-0

16. Shui Y, Li M, Su J, Chen M, Gu X, Guo W. Prognostic and clinicopathological significance of systemic immune-inflammation index in pancreatic cancer: a meta-analysis of 2365 patients. Aging. 2021;13:20585-20597. doi:10.18632/aging.203449

17. Deng Y, Zhao Y, Qin J, et al. Prognostic value of the C-reactive protein/albumin ratio and systemic immune-inflammation index for patients with colorectal liver metastasis undergoing curative resection. Pathol Oncol Res. 2021;27:633480. doi:10.3389/ pore. 2021.633480

18. Matsubara S, Mabuchi S, Takeda Y, Kawahara N, Kobayashi H. Prognostic value of pre-treatment systemic immune-inflammation index in patients with endometrial cancer. PLoS One. 2021;16(5): e0248871. doi:10.1371/journal.pone.0248871

19. Holub K, Busato F, Gouy S, et al. Analysis of systemic inflammatory factors and survival outcomes in endometrial cancer patients staged I-III FIGO and treated with postoperative external radiotherapy. J Clin Med. 2020;9(5):1441. doi:10.3390/jcm9051441

20. Diakos C, Charles K, McMillan D, Clarke S. Cancer-related inflammation and treatment effectiveness. Lancet Oncol. 2014;15(11):e493503. doi:10.1016/S1470-2045(14)70263-3 
21. Crusz S, Balkwill F. Inflammation and cancer: advances and new agents. Nat Rev Clin Oncol. 2015;12(10):584-596. doi:10.1038/ nrclinonc.2015.105

22. Antonioli L, Blandizzi C, Pacher P, Haskó G. Immunity, inflammation and cancer: a leading role for adenosine. Nat Rev Cancer. 2013;13(12):842-857. doi:10.1038/nrc3613

23. Ferrone C, Dranoff G. Dual roles for immunity in gastrointestinal cancers. J Clin Oncol. 2010;28(26):4045-4051. doi:10.1200/ JCO.2010.27.9992

24. Burgess B, Levine B, Taylor R, Kelly M. Preoperative circulating lymphocyte and monocyte counts correlate with patient outcomes in type I and type II endometrial cancer. Reprod Sci. 2020;27 (1):194-203. doi:10.1007/s43032-019-00009-4

25. Aoyama T, Takano M, Miyamoto $M$, et al. Pretreatment neutrophil-to-lymphocyte ratio was a predictor of lymph node metastasis in endometrial cancer patients. Oncology. 2019;96(5):259-267. doi: $10.1159 / 000497184$

26. Moses K, Brandau S. Human neutrophils: their role in cancer and relation to myeloid-derived suppressor cells. Semin Immunol. 2016;28(2):187-196. doi:10.1016/j.smim.2016.03.018

27. Yokoi E, Mabuchi S, Komura N, et al. The role of myeloid-derived suppressor cells in endometrial cancer displaying systemic inflammatory response: clinical and preclinical investigations. Oncoimmunology. 2019;8(12):e1662708. doi:10.1080/2162402X. 2019.1662708
28. Chen H, Wu Q, Zhang Y, et al. Nomograms based on the novel platelet index score predict postoperative prognosis in endometrial cancer. Gynecol Oncol. 2020;158(3):689-697. doi:10.1016/j. ygyno.2020.05.040

29. Mahdi H, Jernigan A, Nutter B, Michener C, Rose P. Lymph node metastasis and pattern of recurrence in clinically early stage endometrial cancer with positive lymphovascular space invasion. J Gynecol Oncol. 2015;26(3):208-213. doi:10.3802/ jgo.2015.26.3.208

30. Hahn H, Lee I, Kim T, et al. Lymphovascular space invasion is highly associated with lymph node metastasis and recurrence in endometrial cancer. Aust N Z J Obstet Gynaecol. 2013;53(3):293-297. doi:10.1111/ajo.12089

31. Jiang P, Huang Y, Tu Y, et al. Combining clinicopathological parameters and molecular indicators to predict lymph node metastasis in endometrioid type endometrial adenocarcinoma. Front Oncol. 2021;11:682925. doi:10.3389/fonc.2021.682925

32. Nithin K, Sridhar M, Srilatha K, Habebullah S. CA 125 is a better marker to differentiate endometrial cancer and abnormal uterine bleeding. Afr Health Sci. 2018;18(4):972-978. doi:10.4314/ahs. v18i4.17

33. Li X, Xu Y, Zhang L. Serum CA153 as biomarker for cancer and noncancer diseases. Prog Mol Biol Transl Sci. 2019;162:265-276.
Journal of Inflammation Research

\section{Publish your work in this journal}

The Journal of Inflammation Research is an international, peerreviewed open-access journal that welcomes laboratory and clinical findings on the molecular basis, cell biology and pharmacology of inflammation including original research, reviews, symposium reports, hypothesis formation and commentaries on: acute/chronic inflammation; mediators of inflammation; cellular processes; molecular

\section{Dovepress}

mechanisms; pharmacology and novel anti-inflammatory drugs; clinical conditions involving inflammation. The manuscript management system is completely online and includes a very quick and fair peerreview system. Visit http://www.dovepress.com/testimonials.php to read real quotes from published authors. 